\title{
Substantial thermoelectric enhancement achieved by manipulating the band structure and dislocations in Ag and La co-doped SnTe
}

\author{
Wenjing $\mathrm{XU}^{a, \dagger}$, Zhongwei ZHANG ${ }^{a, \dagger}$, Chengyan $\mathrm{LIU}^{a,{ }^{*}}$, Jie $\mathrm{GAO}^{a}$, Zhenyuan $\mathrm{YE}^{a}$, \\ Chunguang $\mathrm{CHEN}^{a}$, Ying $\mathrm{PENG}^{a}$, Xiaobo $\mathrm{BAI}^{a}$, Lei MIAO ${ }^{a, b, *}$ \\ ${ }^{a}$ Guangxi Key Laboratory of Information Material, Guangxi Collaborative Innovation Center of Structure and \\ Property for New Energy and Materials, School of Material Science and Engineering, Guilin University of \\ Electronic Technology, Guilin 541004, China \\ ${ }^{b}$ Department of Materials Science and Engineering, SIT Research Laboratories, Innovative Global Program, \\ Faculty of Engineering, Shibaura Institute of Technology, Tokyo 135-8548, Japan
}

Received: February 24, 2021; Revised: March 17, 2021; Accepted: March 31, 2021

(C) The Author(s) 2021.

\begin{abstract}
Eco-friendly SnTe based thermoelectric materials are intensively studied recently as candidates to replace $\mathrm{PbTe}$; yet the thermoelectric performance of $\mathrm{SnTe}$ is suppressed by its intrinsically high carrier concentration and high thermal conductivity. In this work, we confirm that the $\mathrm{Ag}$ and La co-doping can be applied to simultaneously enhance the power factor and reduce the thermal conductivity, contributing to a final promotion of figure of merit. On one hand, the carrier concentration and band offset between valence bands are concurrently reduced, promoting the power factor to a highest value of $\sim 2436 \mu \mathrm{W} \cdot \mathrm{m}^{-1} \cdot \mathrm{K}^{-2}$ at $873 \mathrm{~K}$. On the other hand, lots of dislocations $\left(\sim 3.16 \times 10^{7} \mathrm{~mm}^{-2}\right)$ associated with impurity precipitates are generated, resulting in the decline of thermal conductivity to a minimum value of $1.87 \mathrm{~W} \cdot \mathrm{m}^{-1} \cdot \mathrm{K}^{-1}$ at $873 \mathrm{~K}$. As a result, a substantial thermoelectric performance enhancement up to $z T \approx 1.0$ at $873 \mathrm{~K}$ is obtained for the sample $\mathrm{Sn}_{0.94} \mathrm{Ag}_{0.09} \mathrm{La}_{0.05} \mathrm{Te}$, which is twice that of the pristine $\operatorname{SnTe}(z T \approx 0.49$ at $873 \mathrm{~K})$. This strategy of synergistic manipulation of electronic band and microstructures via introducing rare earth elements could be applied to other systems to improve thermoelectric performance.
\end{abstract}

Keywords: SnTe; band convergence; dislocation; Ag and La co-doping; thermoelectric performance

\section{Introduction}

Clean, reliable, and sustainable energy utilization technologies become more and more important [1].

\footnotetext{
$\dagger$ Wenjing Xu and Zhongwei Zhang contributed equally to this work.

* Corresponding authors.

E-mail: C. Liu, chengyanliu@guet.edu.cn;

L. Miao,miaolei@guet.edu.cn
}

Thermoelectric (TE) materials can provide such technologies to directly convert heat into electricity and vice versa [2-6]. However, the low energy conversion efficiency of TE materials severely restricts their wide range of commercial applications, which can be governed by a dimensionless figure of merit, $z T=\left(S^{2} \sigma / \kappa\right) T$, where $S, \sigma, T$, and $\kappa$ are the Seebeck coefficient, electrical conductivity, absolute temperature, and thermal conductivity, respectively. Among them, $\kappa$ is composed of the electronic thermal conductivity $\left(\kappa_{\mathrm{e}}\right)$ 
and lattice thermal conductivity $\left(\kappa_{1}\right)[7-10]$. Therefore, in order to obtain a high $z T$, it is necessary to optimize the power factor $\left(P F=S^{2} \sigma\right)$ and simultaneously reduce $\kappa$.

However, $S, \sigma$, and $\kappa_{\mathrm{e}}$, which are closely affected by the carrier concentration $(n)$, have a strong coupling relationship with each other, making it difficult to individually optimize a certain parameter without damaging the others [11-14]. Due to the opposite promotion effect of $n$ on $S$ and $\sigma$, the strategy of optimizing TE performance by improving the power factor has become extremely significant, including the introduction of resonance levels $[15,16]$, energy filtering [17], band convergence [18], increasing the band gap [19], etc. In addition, in order to reduce $\kappa_{1}$, many researches focus on introducing point defects [20-22], dislocations [23], nano-precipitation [24,25], and grain boundaries [26] to act as the centers of phonon scattering, thereby effectively preventing phonon transport [27].

Recently, with the development of $\mathrm{Pb}$-free TE materials, SnTe TE materials, which have a band structure similar to $\mathrm{PbTe}$, have intensively attracted attention. Unfortunately, the high carrier concentration $\left(\sim 10^{21} \mathrm{~cm}^{-3}\right)$ caused by excessive $\mathrm{Sn}$ vacancies in SnTe greatly increases $\kappa_{\mathrm{e}}$ and reduces $S$, which seriously affects the TE performance, bringing about a $z T$ value of only about 0.6 at $873 \mathrm{~K}$. Meanwhile, the excessive energy splitting $\left(\Delta E_{\mathrm{v}}\right)$ between light and heavy valence bands is also a reason for its poor TE performance. In response to these problems, various electronic band structure modulation and phonon scattering optimization approaches have been employed recently to enhance the TE performance of SnTe systems. Generally, Sn self-compensation can be used to partially control the carrier concentration $[28,29]$. At the same time, some foreign atomic doping is claimed to effectively converge the light and heavy valence bands, such as $\mathrm{Ag}[18,23]$, $\mathrm{Mn} \mathrm{[30],} \mathrm{Cu}$ [31], and $\mathrm{Mg}$ [19]. In particular, the co-doping of $\mathrm{Ag}$ associated with other elements was reported to effectively tune the electronic band structures for higher TE performance. For example, Banik et al. [15] declared that Ag doping reduces the energy splitting between the light and heavy valence bands while In doping introduces resonance levels inside the valence bands, leading to an improved Seebeck coefficient, and an improved thermoelectric figure of merit, $z T \approx 1$, in $\mathrm{SnAg}_{0.025} \mathrm{In}_{0.025} \mathrm{Te}_{1.05}$ at $856 \mathrm{~K}$. Recently, Mn doping is employed to further converge the valence bands on the basis of $\mathrm{Ag}$ doping. As a consequence, $\Delta E_{\mathrm{v}}$ decreases to $0.1 \mathrm{eV}$ in $\mathrm{Sn}_{0.83} \mathrm{Ag}_{0.03} \mathrm{Mn}_{0.17} \mathrm{Te}$ compared to the value of $0.35 \mathrm{eV}$ in pristine $\mathrm{SnTe}$, and a high power factor of $24.8 \mu \mathrm{W} \cdot \mathrm{m}^{-1} \cdot \mathrm{K}^{-2}$ at $816 \mathrm{~K}$ is attained [32]. On the other hand, ultralow lattice thermal conductivity could be realized by incorporating various microstructures, including dislocations [33,34], precipitates [35], mesoscale grain boundaries [36,37], and point defects [38]. For instance, the all-scale structure defects containing the atomic-scale In doping point defects, the nanoscale $\mathrm{Sb}$ precipitates, and the mesoscale grain boundaries substantially impede the phonon transport so that a minimum value of $\sim 0.44 \mathrm{~W} \cdot \mathrm{m}^{-1} \cdot \mathrm{K}^{-1}$ is realized in $\left(\mathrm{Sn}_{1.06} \mathrm{Te}\right)_{0.95}(\mathrm{InSb})_{0.05}$ at $823 \mathrm{~K}$, leading to a maximum $z T$ value of $\sim 0.84$ [39]. Zheng et al. [18] showed that $\mathrm{Cu}_{2} \mathrm{Te}$ acts as the source of interstitial defects to reduce the lattice thermal conductivity to the amorphous limit, achieving a high $z T$ value (1.4 at $900 \mathrm{~K})$. Motivated by these works, we pay attention to the collaboration with rare earth elements, which are rarely focused on $\mathrm{SnTe}$ systems, on the basis of $\mathrm{Ag}$ doping.

Herein, we obtained a series of $\operatorname{Sn}_{1+\delta} \mathrm{Te}(\delta=0-0.05)$, $\mathrm{Sn}_{1.03-x} \mathrm{Ag}_{x} \mathrm{Te}(x=0.01-0.09)$, and $\mathrm{Sn}_{0.94} \mathrm{Ag}_{0.09} \mathrm{La}_{y} \mathrm{Te}$ $(y=0.01-0.07)$ samples by combining a melting reaction and spark plasma sintering (SPS). The co-doping of Ag and La simultaneously optimizes the transport of carrier and phonon, increasing $S$ while suppressing $\kappa$ over the whole temperature range. The theoretical simulation calculation confirms that the $\mathrm{Ag}$ and $\mathrm{La}$ co-doping weaken the band offset of valence bands and broaden the band gap like a relay race. It is for this reason that the Seebeck coefficient has a jump over the entire temperature range, and no bipolar effect is observed in the $\mathrm{Ag}$ and $\mathrm{La}$ co-doped $\mathrm{SnTe}$, whose carrier concentration is decreased and carrier mobility is increased. In addition, a large number of dislocations associated with impurity precipitates are formed to act as phonon scattering centers, due to the excessive content of foreign dopants that exceed their solubility limit in the host material. As a result, the Seebeck coefficient of the sample $\mathrm{Sn}_{0.94} \mathrm{Ag}_{0.09} \mathrm{La}_{0.05} \mathrm{Te}$ reaches $161 \mu \mathrm{V} \cdot \mathrm{K}^{-1}$, and the thermal conductivity drops to $1.87 \mathrm{~W} \cdot \mathrm{m}^{-1} \cdot \mathrm{K}^{-1}$, which doubles the $z T$ value on the basis of the pristine $\operatorname{SnTe}(z T \approx 0.49)$ to $\sim 1.0$ at $873 \mathrm{~K}$. The improvement of TE performance in this work is comparable to the current research on SnTe based TE materials.

\section{Experimental methods}

For the preparation of high quality samples, $\sim 9 \mathrm{~g}$ 
$\mathrm{Sn}_{1+\delta} \mathrm{Te}(\delta=0-0.05), \mathrm{Sn}_{1.03-x} \mathrm{Ag}_{x} \mathrm{Te}(x=0.01-0.09)$, and $\mathrm{Sn}_{0.94} \mathrm{Ag}_{0.09} \mathrm{La}_{y} \mathrm{Te}(y=0.01-0.07)$ cylinders with diameter of $\sim 12.7 \mathrm{~mm}$ were synthesized by combining a melting reaction and spark plasma sintering (SPS). In detail, Sn (99.9\%, Aladdin), Te (99.9\%, Aladdin), Ag (99.9\%, Aladdin), and La (99.9\%, Aladdin) were weighted with stoichiometric composition and flame-sealed in quartz tubes under a vacuum pressure $(<1 \mathrm{~Pa})$. The tubes were slowly heated to $1273 \mathrm{~K}$ in $12 \mathrm{~h}$ using a programmable furnace, then held for $6 \mathrm{~h}$, and rapidly quenched by ice water. The obtained bulk materials were crushed and milled by hand with an agate mortar, and then transferred into graphite molds lined with carbon papers. Under vacuum condition $(<10 \mathrm{~Pa})$, the samples were quickly transformed into cylinders with relatively high density ( $>97 \%$ ) by SPS under an axial press of $50 \mathrm{MPa}$ at $823 \mathrm{~K}$ for $5 \mathrm{~min}$.

The powders obtained by grinding the SPS sintered bulk materials were analyzed by using an X-ray diffraction measurement (XRD, D8 Advance, Bruker) with $\mathrm{Cu} \mathrm{K \alpha l}$ radiation $(\lambda=1.54 \AA)$ at $40 \mathrm{kV}$ and $40 \mathrm{~mA}$. The microstructures of the as-synthesized samples were characterized by a transmission electron microscopy (TEM, FEI Talos F200X). An electron probe microanalyzer (EPMA, JEOL JXA-8230) was performed to determine the composition distribution. The measurements of electrical conductivity $(\sigma)$ and Seebeck coefficient $(S)$ were performed on a commercial CTA-3 instrument (Beijing Cryoall Science and Technology Co., Ltd., China) in the range of 300-873 K under a low helium atmosphere. The Hall coefficient $\left(R_{\mathrm{H}}\right)$ was measured by the van der Pauw technique (8404, Lakeshore, USA) under a reversible magnetic field of $1.5 \mathrm{~T}$ at room temperature. The carrier concentration ( $n$ ) was estimated through the expression $n=1 /\left(e R_{\mathrm{H}}\right)$, where $e$ is the elementary charge. The Hall carrier mobility $(\mu)$ was calculated from the expression $\mu=\sigma R_{\mathrm{H}}$. The thermal conductivity $(\kappa)$ was determined by the expression $\kappa=D C_{p} \rho$, where $D$ was the thermal diffusivity, $C_{p}$ was the specific heat indexed to the data from Ref. [40], and $\rho$ was the density. The thermal diffusivity $(D)$ was directly measured using the laser flash diffusivity method (Netzsch LFA-467 HT, Germany) at $300-873 \mathrm{~K}$ under an argon flow. The density of the samples was measured using the Archimedes method.

The electronic structures of pristine $\mathrm{SnTe}, \mathrm{Ag}$ doped, $\mathrm{Ag}$ and La co-doped SnTe were simulated using the density functional theory (DFT) in the Vienna Ab initio
Simulation Package (VASP) [41]. The projectoraugmented-wave (PAW) [40] and generalized gradient approximation (GGA) to exchange-correlation term with the Perdew, Burke, and Ernzerhof (PBE) were used in the computations [43]. The cut-off energy of plane-wave set was $400 \mathrm{eV}$. The theoretical models of pristine $\mathrm{SnTe}, \mathrm{Ag}$, La, double Ag, Ag and La co-doped $\mathrm{SnTe}$ were composed of a $3 \times 3 \times 3$ super-cell with 54 atoms. Correspondingly, a $\Gamma$-centered $4 \times 4 \times 4 \mathrm{k}$-mesh was used in the Brillouin zone. Spin-orbit coupling was included in the band structure calculations but not added in the structure relaxation process.

\section{Results and discussion}

To suppress the excess inherent Sn vacancies, we adopted the approach of Sn self-compensation at first, which indeed effectively enhances the figure of merit $z T$ (the detailed data is not shown here). In line with the previous reports [27,28], the sample with $3 \mathrm{~mol} \%$ Sn self-compensation $\left(\mathrm{Sn}_{1.03} \mathrm{Te}\right)$ represents optimized thermoelectric performance in our experiments. On this basis, Ag doping was applied to manipulate the energy separation $\left(\Delta E_{\mathrm{v}}\right)$ between the light and heavy valence bands according to the literatures [15,32]. Among our synthesized $\mathrm{Ag}$ doped samples, $\mathrm{Sn}_{0.94} \mathrm{Ag}_{0.09} \mathrm{Te}$ had the best thermoelectric performance. Therefore, we carried out $\mathrm{La}$ doping in $\mathrm{Sn}_{0.94} \mathrm{Ag}_{0.09} \mathrm{Te}$, namely $\mathrm{Sn}_{0.94} \mathrm{Ag}_{0.09} \mathrm{La}_{y} \mathrm{Te}(y=0-0.07)$.

Figure 1 gives the room-temperature XRD patterns of the pristine $\mathrm{SnTe}$ and the samples $\mathrm{Sn}_{0.94} \mathrm{Ag}_{0.09} \mathrm{La}_{y} \mathrm{Te}$ $(y=0-0.07)$. The main diffraction peaks as shown in Fig. 1(a) could be well matched with the cubic rock-salt phase of SnTe. In addition, weak peaks at $\sim 29.7^{\circ}$ and $\sim 31.1^{\circ}$, which could be indexed to $\mathrm{Ag}_{2} \mathrm{Te}$, can be observed in $\mathrm{Sn}_{0.94} \mathrm{Ag}_{0.09} \mathrm{Te}$ and $\mathrm{Sn}_{0.94} \mathrm{Ag}_{0.09} \mathrm{La}_{0.01} \mathrm{Te}$ (Fig. 1(b)). However, the peak at $\sim 31.1^{\circ}$ disappears, and new peaks at $\sim 29.8^{\circ}$ and $\sim 30.7^{\circ}$ which may be ascribed to lanthanum telluride $\left(\mathrm{La}_{4} \mathrm{Te}_{7}\right.$ and $\left.\mathrm{LaTe}_{3}\right)$ appear after the La doping. Besides, the peak at $\sim 29.8^{\circ}$ strengthens with increasing La content. This phenomenon suggests that the La doping inhibits the formation of $\mathrm{Ag}_{2} \mathrm{Te}$ phase and lanthanum tellurides precipitates generate if the La doping exceeds its low solubility limit $(<1 \mathrm{~mol} \%)$. This is further demonstrated by EPMA of the polished surface, as typically shown in Fig. 2 for $\mathrm{Sn}_{0.94} \mathrm{Ag}_{0.09} \mathrm{La}_{0.05} \mathrm{Te}$. The element mapping discloses that Ag rich (Fig. 2(d)) and La rich (Fig. 2(e)) 

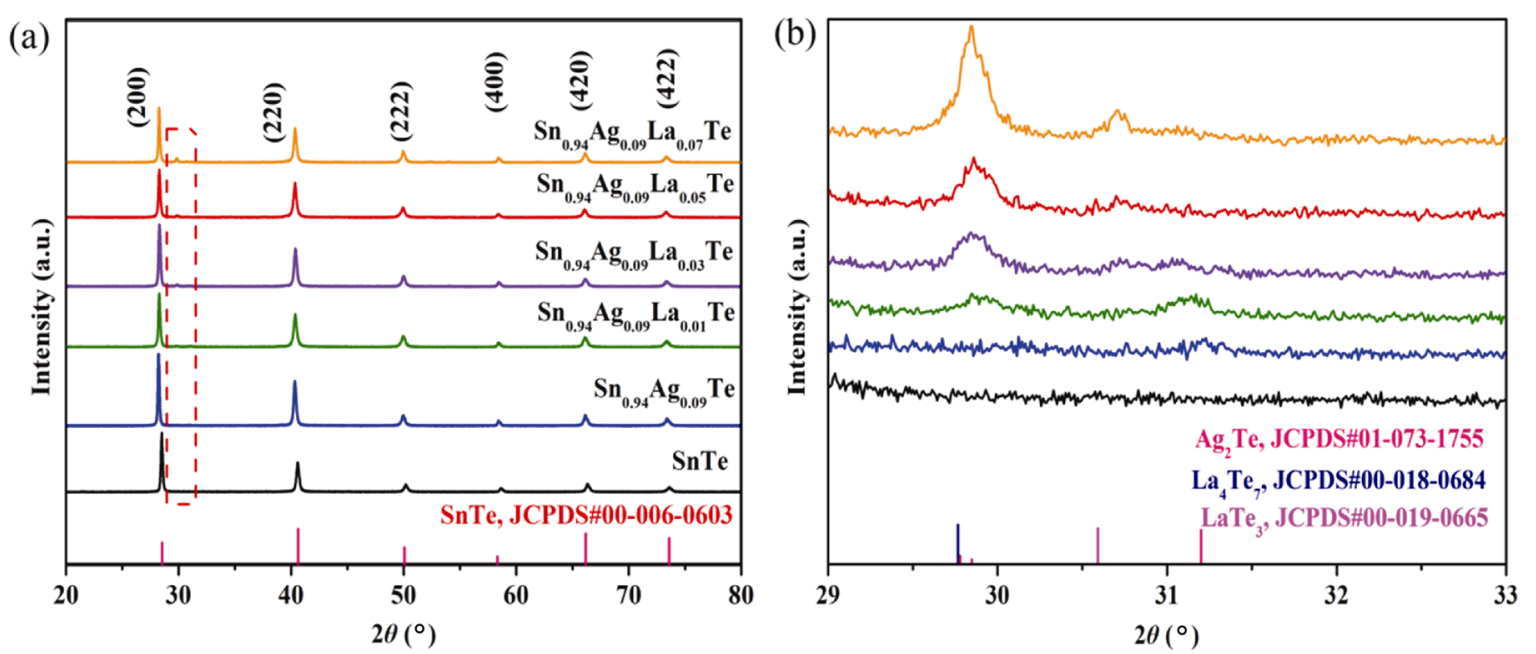

Fig. 1 (a) XRD patterns of the pristine $\mathrm{SnTe}, \mathrm{Sn}_{0.94} \mathrm{Ag}_{0.09} \mathrm{Te}$, and $\mathrm{Sn}_{0.94} \mathrm{Ag}_{0.09} \mathrm{La}_{y} \mathrm{Te}$. (b) Magnified XRD patterns from $29^{\circ}$ to $33^{\circ}$ as marked by a red dotted rectangle in (a).

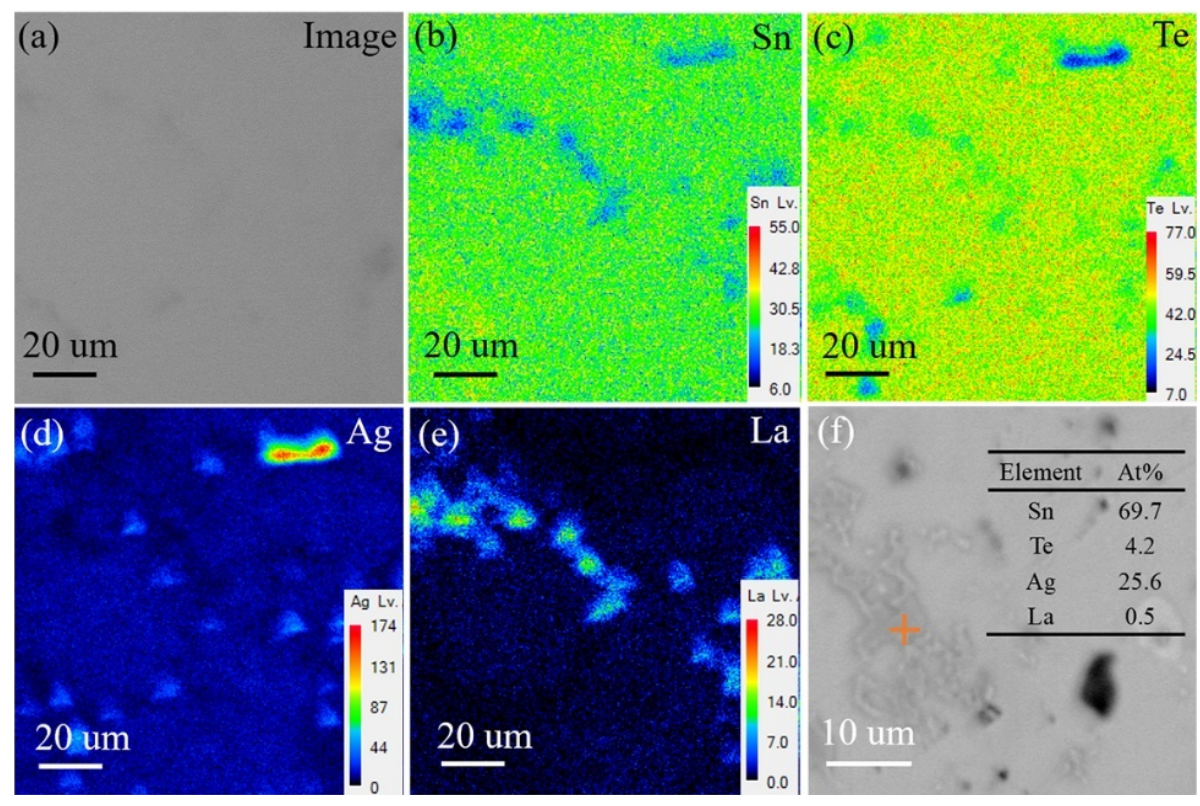

Fig. 2 EPMA characterization of the sample $\mathrm{Sn}_{0.94} \mathrm{Ag}_{0.09} \mathrm{La}_{0.05} \mathrm{Te}$ : (a) a back scattering electron (BSE) image; (b-e) element mapping images of $\mathrm{Sn}, \mathrm{Te}, \mathrm{Ag}$, and La; (f) a BSE image and the composition data for the point marked by a cross.

regions exist in the SnTe matrix (Figs. 2(b) and 2(c)). The point elemental scanning shows that the Ag rich regions are $\mathrm{Ag}-\mathrm{Sn}$ alloys (e.g., point 1 in Fig. 2(f) exhibits that the $\mathrm{Ag} / \mathrm{Sn}$ atomic ratio is 2.72:1) rather than $\mathrm{Ag}_{2} \mathrm{Te}$, though they were not detected by the XRD characterization, possibly due to its relatively low detection sensitivity. Suitable nanoscale impurity precipitates which induce lots of interfaces in matrix are expected to reduce the lattice thermal conductivity through blocking the transport of phonons [39].

Figure 3 represents the electronic transport properties as a function of measurement temperature. As displayed in Fig. 3(a), the electrical conductivity ( $\sigma$ ) of all samples decreases with increasing temperature, indicating that they act as electronic transport behavior of degenerate semiconductors and the bipolar diffusion effect can be ignored. With the help of Sn self-compensation and Ag doping, the electrical conductivity drops over the whole temperature range. Furthermore, the decline of electrical conductivity is relayed by the La doping, especially at low temperatures. For example, the electrical conductivity at $323 \mathrm{~K}$ is decreased from $6.95 \times 10^{5} \mathrm{~S} \cdot \mathrm{m}^{-1}$ (the pristine $\mathrm{SnTe})$ to $5.41 \times 10^{5} \mathrm{~S} \cdot \mathrm{m}^{-1}\left(\mathrm{Sn}_{0.94} \mathrm{Ag}_{0.09} \mathrm{Te}\right)$ by the Sn self-compensation and Ag doping, and further 

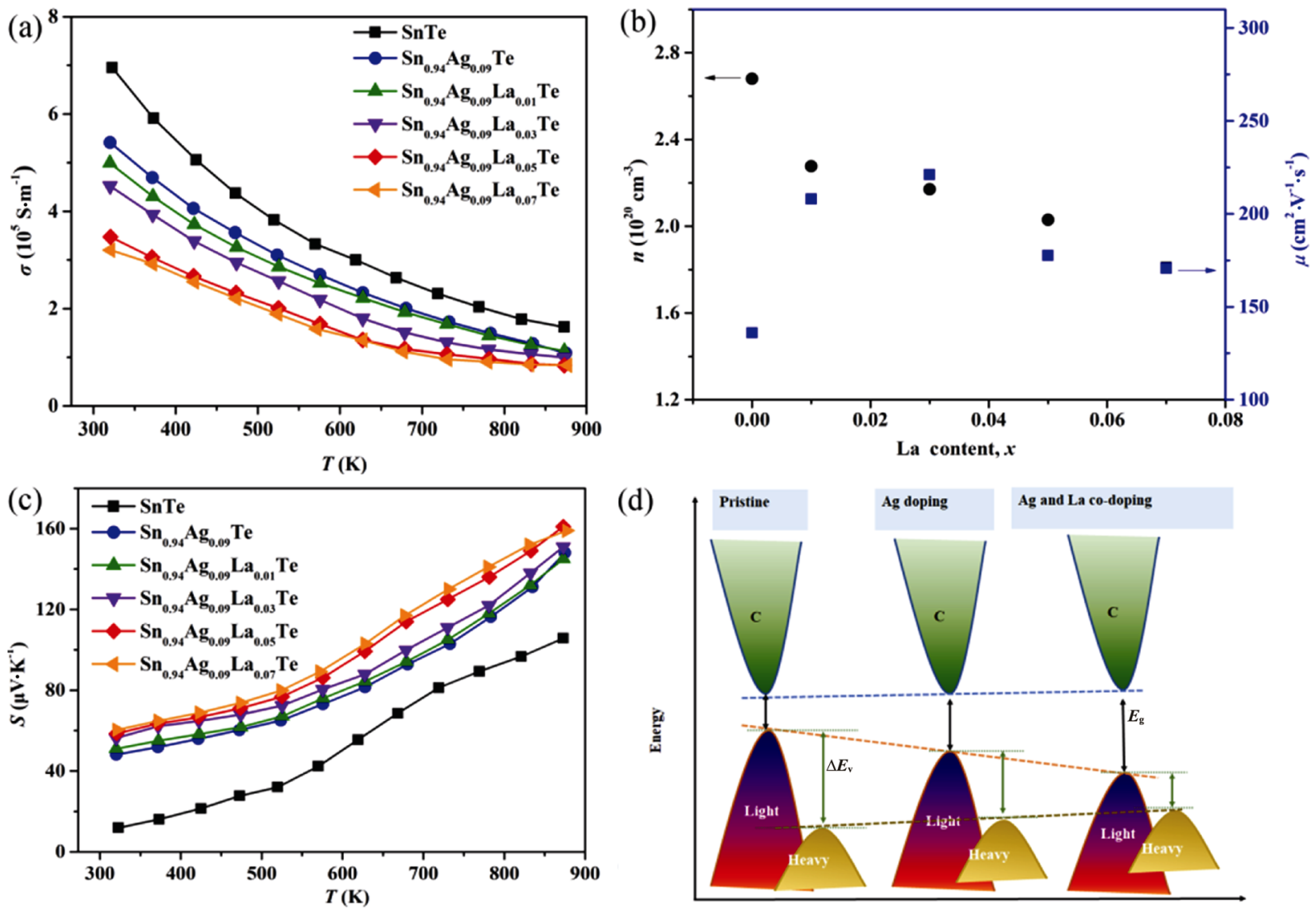

(d)
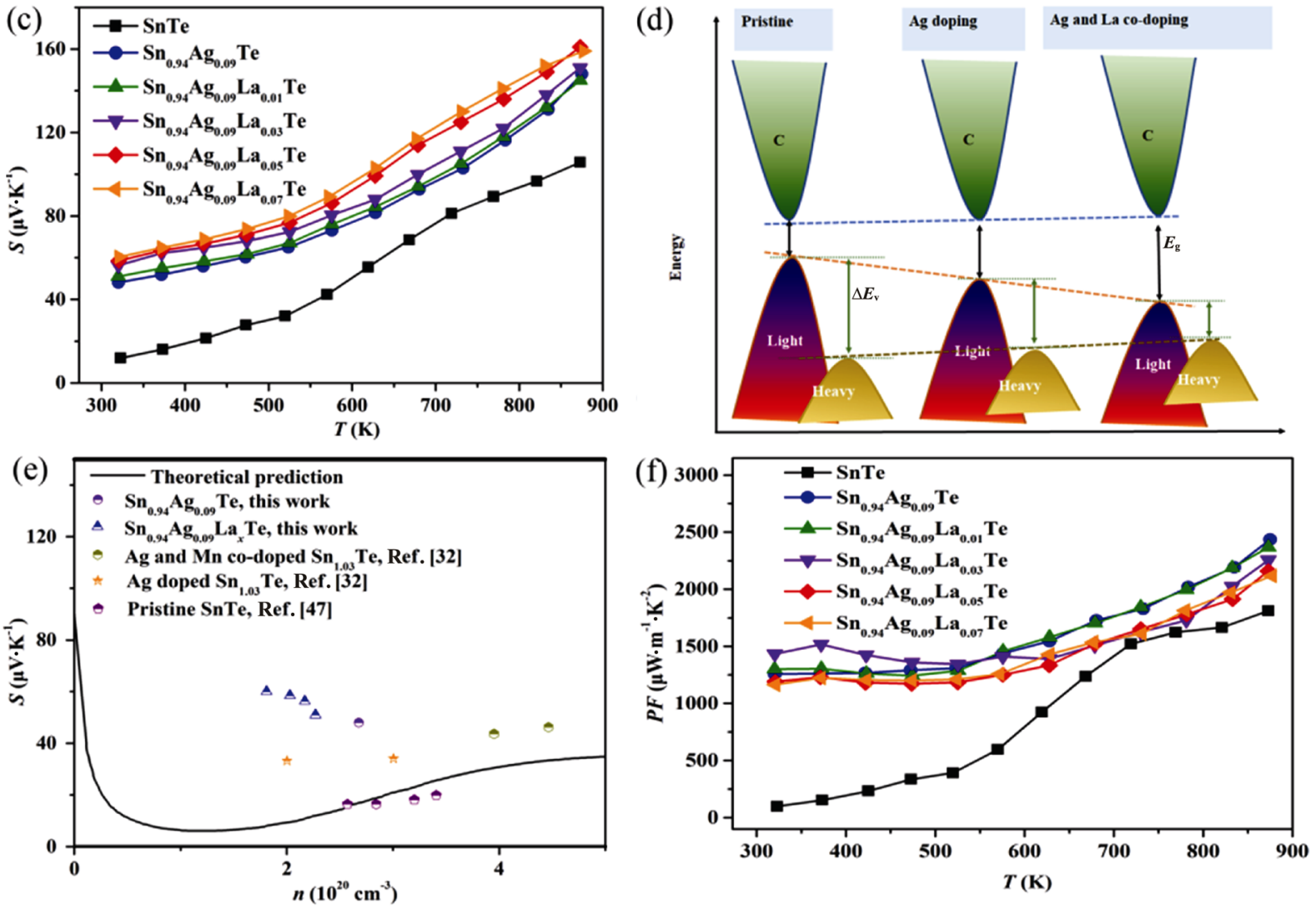

Fig. 3 Electrical properties of the pristine $\mathrm{SnTe}, \mathrm{Sn}_{0.94} \mathrm{Ag}_{0.09} \mathrm{Te}$, and $\mathrm{Sn}_{0.94} \mathrm{Ag}_{0.09} \mathrm{La}_{y} \mathrm{Te}$ : (a) electrical conductivity, (c) Seebeck coefficient, and (f) power factor as a function of temperature; (b) carrier concentration and mobility as a function of the La content $y$ at room temperature; (d) schematic presentation of band convergence and widening band gap; (e) Seebeck coefficient as a function of carrier concentration at room temperature. The solid line is a Pisarenko plot at $300 \mathrm{~K}$ computed by using a two-valence-band model. The experimental data of pristine $\mathrm{SnTe}$, Ag doped $\mathrm{Sn}_{1.03} \mathrm{Te}$, and $\mathrm{Ag}$ and $\mathrm{Mn}$ co-doped $\mathrm{Sn}_{1.03} \mathrm{Te}$ are inserted for comparison.

reduced to $3.47 \times 10^{5} \mathrm{~S} \cdot \mathrm{m}^{-1}\left(\mathrm{Sn}_{0.94} \mathrm{Ag}_{0.09} \mathrm{La}_{0.05} \mathrm{Te}\right)$ through the La doping. At $873 \mathrm{~K}$, the electrical conductivity is depressed from $1.62 \times 10^{5}$ to $0.83 \times 10^{5} \mathrm{~S} \cdot \mathrm{m}^{-1}$ with the co-action of Sn self-compensation, $\mathrm{Ag}$ and La co-doping. It is well known that $\mathrm{Sn}$ self-compensation could suppress the hole concentration, leading to the decline of electrical conductivity $[28,29]$. To disclose the physical mechanism of electrical variation caused by the La doping, we obtained the carrier concentration $(n)$ and mobility $(\mu)$ depending on the content of La doping by the Hall effect measurement at room temperature. From Fig. 3(b), we can conclude that the carrier concentration gradually decreases with the rise of La content while the carrier mobility increases at 
first and then reverses at $3 \mathrm{~mol} \% \mathrm{La}$ doping. Exactly, the carrier concentration is moderately reduced from $2.68 \times 10^{20} \mathrm{~cm}^{-3}$ for $\mathrm{Sn}_{0.94} \mathrm{Ag}_{0.09}$ Te to $1.81 \times 10^{20} \mathrm{~cm}^{-3}$ for $\mathrm{Sn}_{0.94} \mathrm{Ag}_{0.09} \mathrm{La}_{0.05} \mathrm{Te}$. The substitution of $\mathrm{La}^{3+}$ on the cationic lattice sites $\left(\mathrm{Sn}^{2+}\right)$, which could serve as a donor type dopant in SnTe matrix, may be responsible for the reduction of carrier concentration. However, complete participation of $1 \mathrm{~mol} \% \mathrm{La}$ atoms into cationic lattice sites should theoretically produce a reduction of $3.2 \times 10^{20} \mathrm{~cm}^{-3}$ on carrier concentration, which suggests that only a small part of atomic La could enter into the cationic lattice sites $(<1 \mathrm{~mol} \%)$. This is in accordance with the above XRD results, which exposes that lanthanum telluride precipitates formed even introduced only $1 \mathrm{~mol} \%$ La. Meanwhile, the carrier mobility is increased from 136 to $208 \mathrm{~cm}^{2} \cdot \mathrm{V}^{-1} \cdot \mathrm{s}^{-1}$ through a slight La doping ( $1 \mathrm{~mol} \%$ ), possibly due to the fact that a portion of Sn vacancies may be filled up by La atoms. In fact, the promotion of carrier mobility by filling up the cationic vacancies is also reported in $\mathrm{Cu}_{2} \mathrm{Te}$-alloyed $\mathrm{PbTe}$ [44]. Nevertheless, the low solubility limit of $\mathrm{La}$ in the SnTe lattice $(<1 \mathrm{~mol} \%)$ prohibits the further enhancement of carrier mobility and even the excessive La doping decreases the carrier mobility. It is reasonable to consider that the interfaces between lanthanum telluride precipitates and SnTe matrix induced by excessive La doping may play electron scattering centers, so that the carrier mobility is reduced to $170 \mathrm{~cm}^{2} \cdot \mathrm{V}^{-1} \cdot \mathrm{s}^{-1}$ for the sample $\mathrm{Sn}_{0.94} \mathrm{Ag}_{0.09} \mathrm{La}_{0.07} \mathrm{Te}$. In a word, the downward change of carrier concentration overcomes the improvement of carrier mobility, resulting in the reduction of electrical conductivity as evaluated by the formula $\sigma=n e \mu$.

Figure 3(c) shows that the Seebeck coefficient of all samples is positive value, indicating that the majority carriers are holes. Besides, it increases with rising the measurement temperature, which could be ascribed to the gradual participation of heavy valence bands for electron transport at elevated temperatures. Furthermore, the integrated effect of Sn self-compensation, $\mathrm{Ag}$ and La co-doping indeed increases the Seebeck coefficient over the whole temperature range. For instance, the Seebeck coefficient at room temperature is $12 \mu \mathrm{V} \cdot \mathrm{K}^{-1}$ for the pristine $\mathrm{SnTe}$, which increases to $48 \mu \mathrm{V} \cdot \mathrm{K}^{-1}$ for the sample $\mathrm{Sn}_{0.94} \mathrm{Ag}_{0.09} \mathrm{Te}$, and further to $59 \mu \mathrm{V} \cdot \mathrm{K}^{-1}$ for the sample $\mathrm{Sn}_{0.94} \mathrm{Ag}_{0.09} \mathrm{La}_{0.05}$ Te. A maximum value of $161 \mu \mathrm{V} \cdot \mathrm{K}^{-1}$ was obtained for the sample $\mathrm{Sn}_{0.94} \mathrm{Ag}_{0.09} \mathrm{La}_{0.05} \mathrm{Te}$ at $873 \mathrm{~K}$. It is expected that the convergence of light bands and heavy bands as schematically shown in Fig. 3(d) should increase the Seebeck coefficient, according to the relationship [45]:

$$
S=\frac{8 \pi k_{\mathrm{B}}^{2}}{3 e h^{2}} N_{\mathrm{V}}^{2 / 3} m_{\mathrm{b}}^{*} T\left(\frac{\pi}{3 n}\right)^{2 / 3}
$$

where $k_{\mathrm{B}}, h, N_{\mathrm{V}}$, and $m_{\mathrm{b}}^{*}$ are the Boltzmann constant, the Plank constant, the valley degeneracy, and the effective mass of each valley, respectively. To clarify the origin of the boosted Seebeck coefficient, the Pisarenko plot calculated on the basis of a two-valence-band model is used as a reference line [46], where similar reported data is also cited for a comparison [32,47]. As shown in Fig. 3(e), the Seebeck coefficient of the samples $\mathrm{Sn}_{0.94} \mathrm{Ag}_{0.09} \mathrm{Te}$ and $\mathrm{Sn}_{0.94} \mathrm{Ag}_{0.09} \mathrm{La}_{y} \mathrm{Te}$ obviously stays above the Pisarenko line. Theoretical electronic band calculation is applied to prove that the band alignment induced by the Ag and La co-doping is the main reason holding out the Seebeck coefficient enhancement.

Figure 4 shows the band structures of $\mathrm{Sn}_{27} \mathrm{Te}_{27}$, $\mathrm{Sn}_{25} \mathrm{Ag}_{2} \mathrm{Te}_{27}, \mathrm{Sn}_{26} \mathrm{La}_{1} \mathrm{Te}_{27}$, and $\mathrm{Sn}_{25} \mathrm{Ag}_{1} \mathrm{La}_{1} \mathrm{Te}_{27}$ calculated by the first-principles simulation, where the effect of spin-orbit coupling (SOC) is taken into consideration since $\mathrm{Te}$ is a heavy element and its $5 \mathrm{p}$ orbital could contribute much to the valence band. The direct band gap $\left(E_{\mathrm{g}}\right)$ of $\mathrm{Sn}_{27} \mathrm{Te}_{27}$ super-cell standing for the pristine $\mathrm{SnTe}$ is $0.1 \mathrm{eV}$ at L point (Fig. 4(a)), which is in line with the previous calculation $(0.11 \mathrm{eV}$ [15]). In contrast with the pristine $\mathrm{SnTe}$, the $\mathrm{Ag}$ doping (Fig. 4(b), $\mathrm{Sn}_{25} \mathrm{Ag}_{2} \mathrm{Te}_{27}$ ), La doping (Fig. 4(c), $\mathrm{Sn}_{26} \mathrm{La}_{1} \mathrm{Te}_{27}$ ), and $\mathrm{Ag}$ and $\mathrm{La}$ co-doping (Fig. 4(d), $\mathrm{Sn}_{25} \mathrm{Ag}_{1} \mathrm{La}_{1} \mathrm{Te}_{27}$ ) effectively broaden the band gap to $0.11,0.13$, and $0.27 \mathrm{eV}$, respectively. The narrow band gap in SnTe may cause the bipolar diffusion effect if the carrier concentration is reduced, which could be reflected by a possible drop of Seebeck coefficient at high temperature, while the increased band gap should eliminate this problem. The disappearance of inflection point for the Seebeck coefficient in our experiments qualitatively supports the theoretical calculation. Moreover, the introduction of $\mathrm{Ag}$ and La synergistically decreases the band offset $\Delta E_{\mathrm{v}}$. Particularly, the Ag and La co-doping strongly weakens the band separation from $0.25 \mathrm{eV}$ for $\mathrm{Sn}_{27} \mathrm{Te}_{27}$ to $0.08 \mathrm{eV}$ for $\mathrm{Sn}_{25} \mathrm{Ag}_{1} \mathrm{La}_{1} \mathrm{Te}_{27}$, and that is why we collected the superior Seebeck coefficient in the samples $\mathrm{Sn}_{0.94} \mathrm{Ag}_{0.09} \mathrm{La}_{y} \mathrm{Te}$.

Thanks to the greatly improved Seebeck coefficient, the power factor (Fig. 3(f)) is elevated over the entire temperature range, and a highest value of $\sim 2436$ $\mu \mathrm{W} \cdot \mathrm{m}^{-1} \cdot \mathrm{K}^{-2}$ at $873 \mathrm{~K}$ is obtained for the sample 
$\mathrm{Sn}_{0.94} \mathrm{Ag}_{0.09} \mathrm{La}_{0.01} \mathrm{Te}$. This is comparable to the best results reported in $\mathrm{AgInTe}_{2}$-alloyed $\mathrm{SnTe}, \mathrm{HgTe}$-alloyed $\mathrm{Sn}_{0.98} \mathrm{Bi}_{0.02} \mathrm{Te}$, and In, Cd co-doped SnTe $[15,16,48]$.
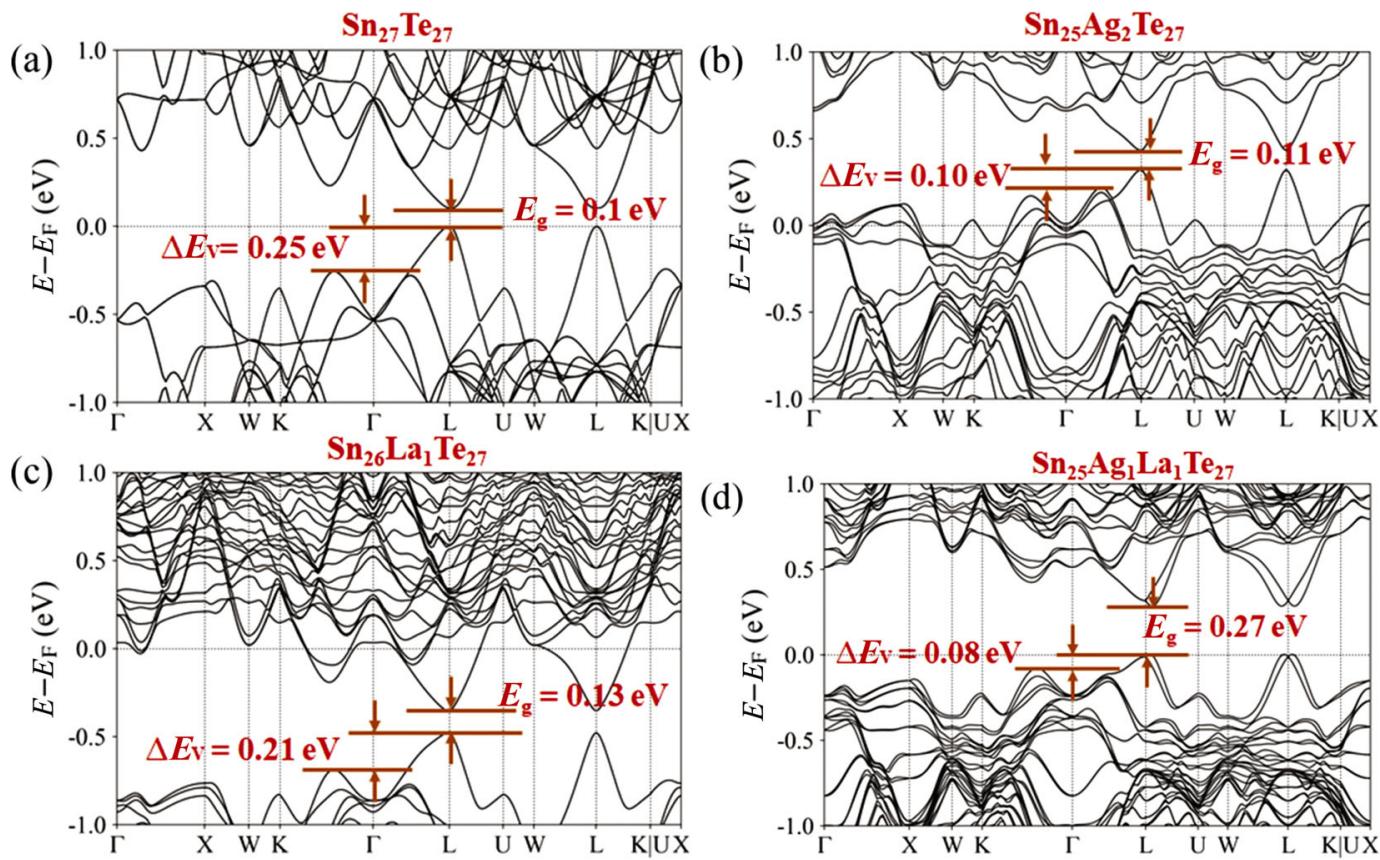

Fig. 4 Band structures of the SnTe system: (a) $\mathrm{Sn}_{27} \mathrm{Te}_{27}$, (b) $\mathrm{Sn}_{25} \mathrm{Ag}_{2} \mathrm{Te}_{27}$, (c) $\mathrm{Sn}_{26} \mathrm{La}_{1} \mathrm{Te}_{27}$, (d) $\mathrm{Sn}_{25} \mathrm{Ag}_{1} \mathrm{La}_{1} \mathrm{Te}_{27}$.
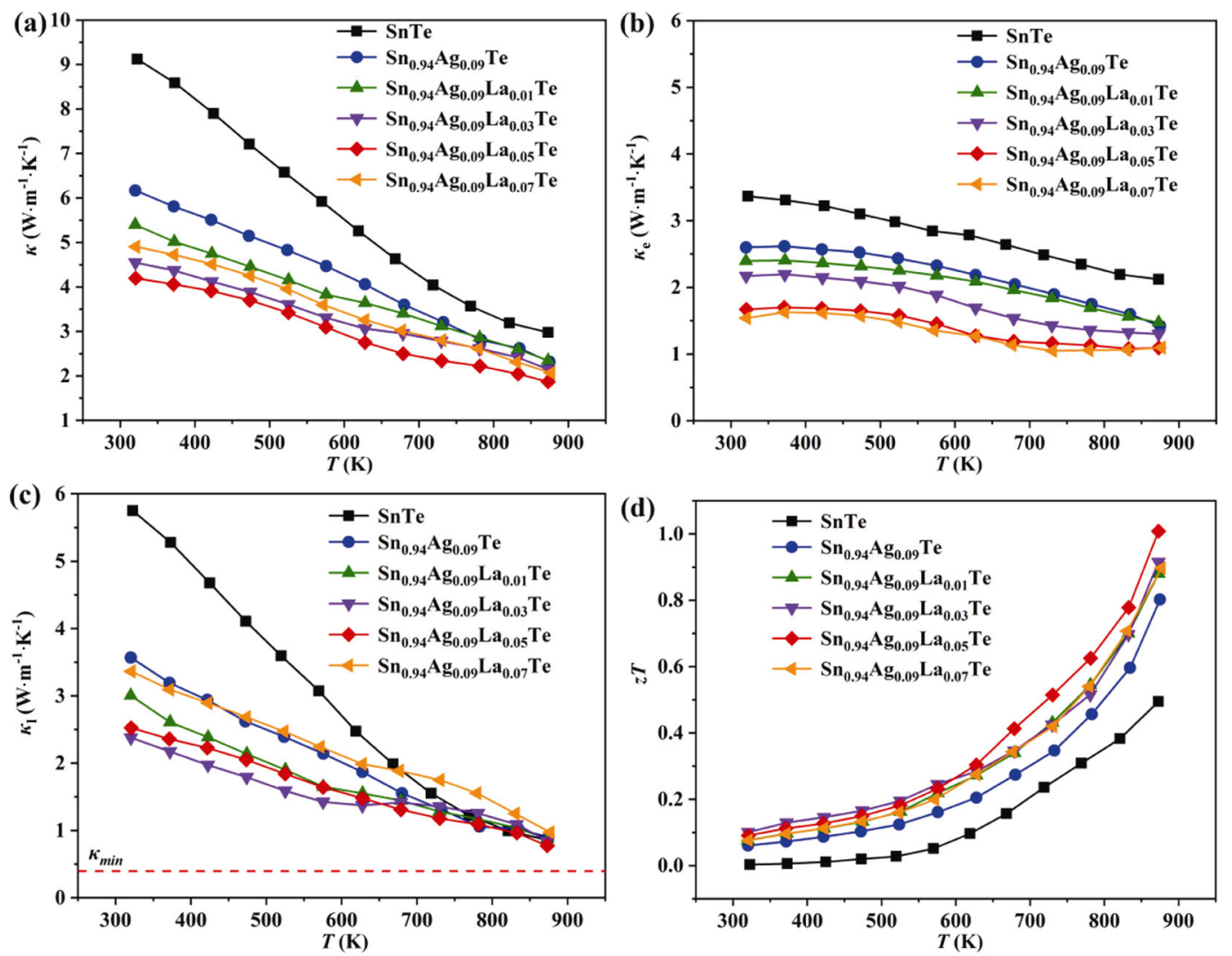

Fig. 5 Temperature-dependent (a) total thermal conductivity, (b) carrier thermal conductivity, (c) lattice thermal conductivity, and (d) $z T$ value of the pristine $\mathrm{SnTe}, \mathrm{Sn}_{0.94} \mathrm{Ag}_{0.09} \mathrm{Te}$, and $\mathrm{Sn}_{0.94} \mathrm{Ag}_{0.09} \mathrm{La}_{y} \mathrm{Te}$. 
figure, we could claim that the Ag and La co-doping worked together to depress the total thermal conductivity over the whole temperature range. At $323 \mathrm{~K}$, the total thermal conductivity is remarkably reduced from $9.12 \mathrm{~W} \cdot \mathrm{m}^{-1} \cdot \mathrm{K}^{-1}$ for the pristine SnTe to $4.19 \mathrm{~W} \cdot \mathrm{m}^{-1} \cdot \mathrm{K}^{-1}$ for the sample $\mathrm{Sn}_{0.94} \mathrm{Ag}_{0.09} \mathrm{La}_{0.05} \mathrm{Te}$. As a result, a minimum value of $1.87 \mathrm{~W} \cdot \mathrm{m}^{-1} \cdot \mathrm{K}^{-1} \quad\left(\mathrm{Sn}_{0.94} \mathrm{Ag}_{0.09} \mathrm{La}_{0.05} \mathrm{Te}\right)$ is obtained at $873 \mathrm{~K}$. The separated analysis of carrier and phonon contribution parts could provide a clear identification for the reduction of thermal conductivity. The carrier thermal conductivity $\left(\kappa_{\mathrm{e}}\right)$ is estimated by using the equation $\kappa_{\mathrm{e}}=L \sigma T$ with the most used $L$ of $1.5 \times 10^{-8} \mathrm{~W} \cdot \Omega \cdot \mathrm{K}^{-2}$, and the lattice thermal conductivity is obtained from $\kappa_{1}=\kappa-\kappa_{\mathrm{e}}$. As shown in Fig. 5(b), the carrier thermal conductivity is effectively decreased with the help of $\mathrm{Ag}$ and $\mathrm{La}$ co-doping as expected since the electrical conductivity is diminished. Correspondingly, the lattice thermal conductivity as a function of temperature is plotted in Fig. 5(c), which decreases continuously with increasing temperature. In surprise, the $\mathrm{Ag}$ and $\mathrm{La}$ co-doping greatly blocks the phonon transport at low temperature. For instance, the lattice thermal conductivity is decreased over one time, from $5.73 \mathrm{~W} \cdot \mathrm{m}^{-1} \cdot \mathrm{K}^{-1}$ for the pristine $\mathrm{SnTe}$ to $2.53 \mathrm{~W} \cdot \mathrm{m}^{-1} \cdot \mathrm{K}^{-1}$ for the sample $\mathrm{Sn}_{0.94} \mathrm{Ag}_{0.09} \mathrm{La}_{0.05} \mathrm{Te}$. On the other hand, the corresponding variation of lattice thermal conductivity at high temperature is nearly indistinguishable, though a minimum value of 0.77 $\mathrm{W} \cdot \mathrm{m}^{-1} \cdot \mathrm{K}^{-1}$ close to the theoretical minimum thermal conductivity $\left(\kappa_{\min } \approx 0.4 \mathrm{~W} \cdot \mathrm{m}^{-1} \cdot \mathrm{K}^{-1}\right)$ of SnTe is obtained for the sample $\mathrm{Sn}_{0.94} \mathrm{Ag}_{0.09} \mathrm{La}_{0.05} \mathrm{Te}$ at $873 \mathrm{~K}$ [49].

To understand the reduced lattice thermal conductivity attained in the sample $\mathrm{Sn}_{0.94} \mathrm{Ag}_{0.09} \mathrm{La}_{0.05} \mathrm{Te}$, its microstructural information is characterized by TEM (Fig. 6). Figure 6(a) reveals that there exists lots of dislocations (marked by the red arrows). Besides, impurity precipitates and grain boundaries are other important microstructures, as identified by the purple dotted rhombus and yellow straight line, respectively. As shown in Fig. 6(b), the crystal plane spacing of $\sim 6.67 \AA$ for the impurity precipitate could be indexed to the (004) plane of $\mathrm{LaTe}_{3}$, while the matrix is the
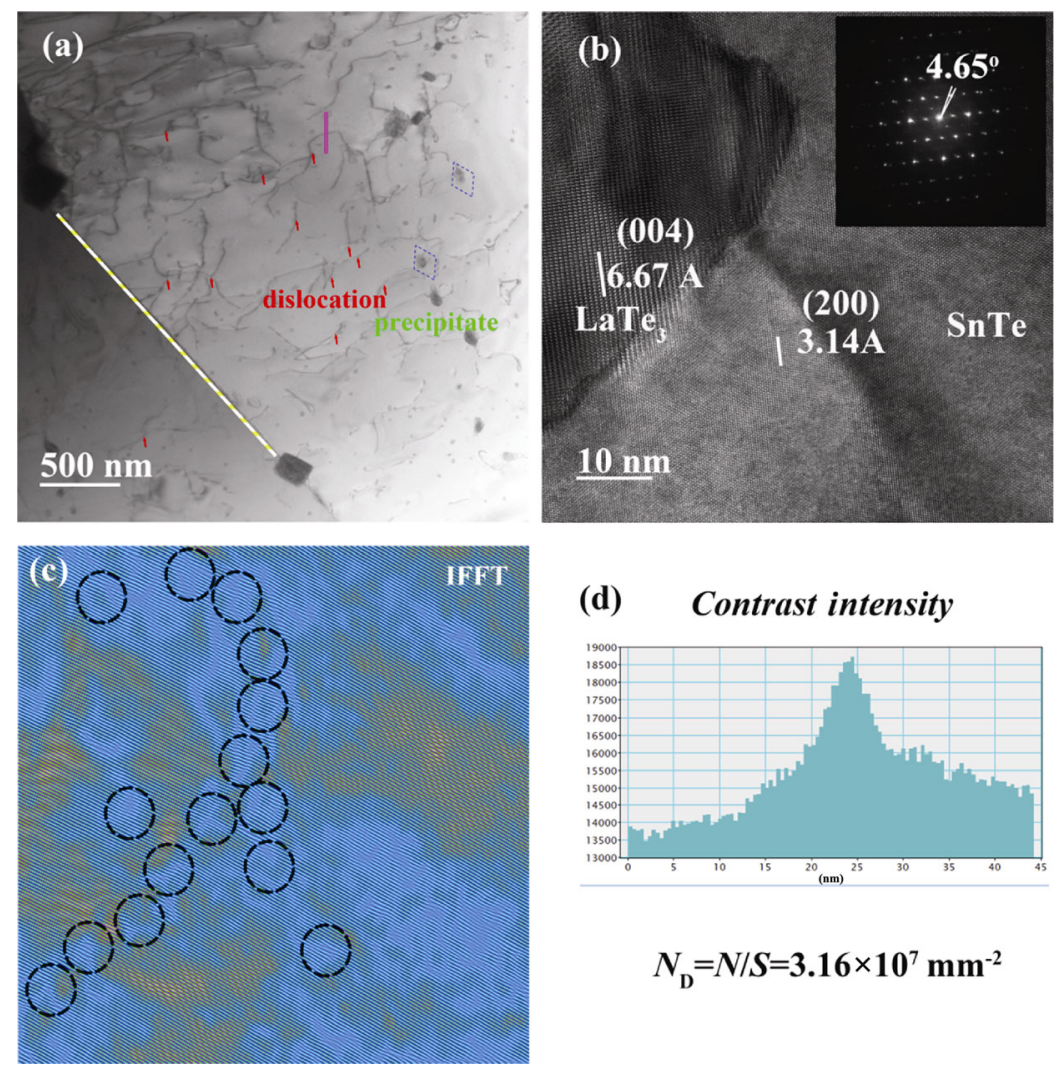

(d) Contrast intensity

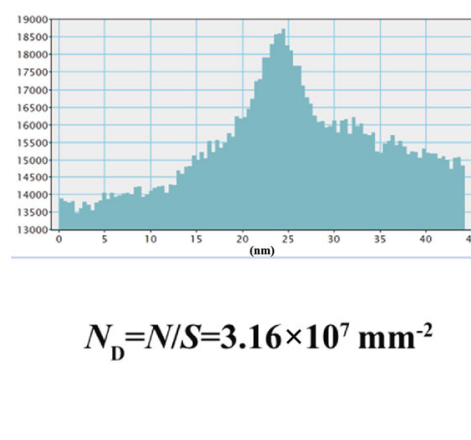

Fig. 6 (a) Low magnified TEM image, (b) high resolution TEM image focusing on the interface between impurity precipitate and matrix, and (c) its corresponding inverse fast Fourier transform (IFFT) pattern of the sample $\mathrm{Sn}_{0.94} \mathrm{Ag}_{0.09} \mathrm{La}_{0.05} \mathrm{Te}$; (d) density of dislocation $\left(N_{\mathrm{D}}\right)$ calculated by dividing the area $(S$, the image shown in (a)) to the amount of dislocation $(N)$ according to the contrast intensity. 
SnTe phase because of the well match between the crystal plane spacing of $\sim 3.14 \AA$ and (200) plane of SnTe. In addition, the observation of interface between impurity precipitates and matrix unveils that the mismatch of (220) crystal planes between $\mathrm{LaTe}_{3}$ and $\mathrm{SnTe}$ is $\sim 4.65^{\circ}$ (the inset in Fig. 6(b)). This semi-coherent heterogeneous grain boundary may strongly prohibit the phonon transport while provide weak influence for the transport of carriers [50]. The IFFT pattern as given in Fig. 6(c) demonstrates that there exist lots of dislocations (marked by the dotted circles) at the semi-coherent heterogeneous grain boundary. In fact, the grain boundary could also be better described as a collection of dislocations rather than perfectly scattering interfaces for phonons [51]. Collectively, $N_{\mathrm{d}}$ is evaluated to be $\sim 3.16 \times 10^{7} \mathrm{~mm}^{-2}$ through contrast intensity (Fig. 6(d)), which is comparable to the level of melt-centrifuged $(\mathrm{Bi}, \mathrm{Sb})_{2} \mathrm{Te}_{3}$ [52]. Mion et al. [53] showed that dislocation density above $10^{5} \mathrm{~mm}^{-2}$ has a significant effect on the lattice thermal conductivity and thus we believe that the dislocation density in the sample $\mathrm{Sn}_{0.94} \mathrm{Ag}_{0.09} \mathrm{La}_{0.05} \mathrm{Te}$ is of an appropriate order of magnitude to lower the lattice thermal conductivity. Note that further increasing in La content could result in formation of excessive impurity aggregations with large size and high thermal conductivity (e.g., $\mathrm{Ag}-\mathrm{Sn}$ alloys), which may be responsible for the elevation of $\kappa_{1}$ on the sample $\mathrm{Sn}_{0.94} \mathrm{Ag}_{0.09} \mathrm{La}_{0.07} \mathrm{Te}$.

The simultaneous optimization of carrier and phonon transport promotes the figure of merit $z T$. As given in Fig. 5(d), the Ag and La co-doping enhances the $z T$ value over the whole temperature range. Finally, a maximum value of $\sim 1.0$ is achieved for the sample $\mathrm{Sn}_{0.94} \mathrm{Ag}_{0.09} \mathrm{La}_{0.05} \mathrm{Te}$ at $873 \mathrm{~K}$, about one time enhancement in comparison with that of the pristine SnTe ( 0.49). This promotion rivals most of the previous works for the SnTe based TE materials [2,38].

\section{Conclusions}

In summary, the co-doping of $\mathrm{Ag}$ and $\mathrm{La}$ optimizes the band structure (valence band convergence and widening band gap) to enhance the Seebeck coefficient together with introducing an appropriate density of dislocations as phonon scattering centers. As a consequence, our results show that the SnTe system co-doped with $\mathrm{Ag}$ and $\mathrm{La}$ achieves a satisfactory power factor $(\sim 2436$ $\mu \mathrm{W} \cdot \mathrm{m}^{-1} \cdot \mathrm{K}^{-2}$ at $873 \mathrm{~K}$ ), and its thermal conductivity is significantly reduced to $1.87 \mathrm{~W} \cdot \mathrm{m}^{-1} \cdot \mathrm{K}^{-1}$ at $873 \mathrm{~K}$. Ultimately, the thermoelectric performance of the sample $\mathrm{Sn}_{0.94} \mathrm{Ag}_{0.09} \mathrm{La}_{0.05} \mathrm{Te}(z T \approx 1.0)$ is twice that of the pristine $\operatorname{SnTe}(z T \approx 0.49)$ at $873 \mathrm{~K}$. This work proves that dense dislocations of appropriate magnitude and electronic band engineering can be simultaneously generated by introducing rare earth element $\mathrm{La}$ to effectively improve the thermoelectric properties of $\mathrm{SnTe}$, which has a certain guiding effect on the research of thermoelectric materials with similar crystal structure.

\section{Acknowledgements}

This work was supported by National Natural Science Foundation of China (Grant Nos. 51801040, 51961011, 51772056) and Guangxi Natural Science Foundation of China (Grant Nos. 2020GXNSFAA159111, AD20159006, 2018GXNSFAA294135, 2018JJA160257, and 2019GXNSFBA245028).

\section{References}

[1] Chu S, Majumdar A. Opportunities and challenges for a sustainable energy future. Nature 2012, 488: 294-303.

[2] Moshwan R, Yang L, Zou J, et al. Eco-friendly SnTe thermoelectric materials: Progress and future challenges. Adv Funct Mater 2017, 27: 1703278.

[3] Zhang Q, Liao B, Lan Y, et al. High thermoelectric performance by resonant dopant indium in nanostructured SnTe. PNAS 2013, 110: 13261-13266.

[4] Zhang X, Zhao LD. Thermoelectric materials: Energy conversion between heat and electricity. $J$ Materiomics 2015, 1: 92-105.

[5] Shi X, Chen L, Uher C. Recent advances in high-performance bulk thermoelectric materials. Int Mater Rev 2016, 61: 379-415.

[6] Shi XL, Ai X, Zhang QH, et al. Enhanced thermoelectric properties of hydrothermally synthesized n-type Se\&Lucodoped $\mathrm{Bi}_{2} \mathrm{Te}_{3}$. J Adv Ceram 2020, 9: 424-431.

[7] Shi XL, Tao XY, Zou J, et al. High-performance thermoelectric SnSe: Aqueous synthesis, innovations, and challenges. Adv Sci 2020, 7: 1902923.

[8] Tan GJ, Zhao LD, Kanatzidis MG. Rationally designing high-performance bulk thermoelectric materials. Chem Rev 2016, 116: 12123-12149.

[9] Hochbaum AI, Chen R, Delgado RD, et al. Enhanced thermoelectric performance of rough silicon nanowires. Nature 2008, 451: 163-167.

[10] Zhang R, Pei J, Han ZJ, et al. Optimal performance of $\mathrm{Cu}_{1.8} \mathrm{~S}_{1-x} \mathrm{Te}_{x}$ thermoelectric materials fabricated via high-pressure process at room temperature. $J A d v$ Ceram 2020, 9: 535-543. 
[11] Li XT, Liu JZ, Li S, et al. Synergistic band convergence and endotaxial nanostructuring: Achieving ultralow lattice thermal conductivity and high figure of merit in eco-friendly SnTe. Nano Energy 2020, 67: 104261.

[12] Wang XX, Liu CY, Chen JL, et al. Synergistically optimizing the thermoelectric properties of polycrystalline $\mathrm{Ag}_{8} \mathrm{SnSe}_{6}$ by introducing additional Sn. CrystEngComm 2020, 22: 248-256.

[13] Zhang L, Liu YC, Tan TT, et al. Thermoelectric performance enhancement by manipulation of $\mathrm{Sr} / \mathrm{Ti}$ doping in two sublayers of $\mathrm{Ca}_{3} \mathrm{Co}_{4} \mathrm{O}_{9}$. J Adv Ceram 2020, 9: 769-781.

[14] Zheng YP, Zou MC, Zhang W, et al. Electrical and thermal transport behaviours of high-entropy perovskite thermoelectric oxides. $J$ Adv Ceram 2021, 10: 377-384.

[15] Banik A, Shenoy US, Saha S, et al. High power factor and enhanced thermoelectric performance of $\mathrm{SnTe}^{-\mathrm{AgInTe}} \mathrm{I}_{2}$ : Synergistic effect of resonance level and valence band convergence. J Am Chem Soc 2016, 138: 13068-13075.

[16] Tan GJ, Shi FY, Hao SQ, et al. Codoping in SnTe: Enhancement of thermoelectric performance through synergy of resonance levels and band convergence. $J \mathrm{Am}$ Chem Soc 2015, 137: 5100-5112.

[17] Zhou ZW, Yang JY, Jiang QH, et al. Thermoelectric performance of $\mathrm{SnTe}$ with $\mathrm{ZnO}$ carrier compensation, energy filtering, and multiscale phonon scattering. $J \mathrm{Am}$ Ceram Soc 2017, 100: 5723-5730.

[18] Zheng LL, Li W, Lin SQ, et al. Interstitial defects improving thermoelectric $\mathrm{SnTe}$ in addition to band convergence. ACS Energy Lett 2017, 2: 563-568.

[19] Banik A, Shenoy US, Anand S, et al. Mg alloying in SnTe facilitates valence band convergence and optimizes thermoelectric properties. Chem Mater 2015, 27: 581-587.

[20] Tang J, Yao Z, Chen Z, et al. Maximization of transporting bands for high-performance SnTe alloy thermoelectrics. Mater Today Phys 2019, 9: 100091.

[21] Liu XF, Zhang B, Chen Y, et al. Achieving enhanced thermoelectric performance in $(\mathrm{SnTe})_{1-x}\left(\mathrm{Sb}_{2} \mathrm{Te}_{3}\right)_{x}$ and $(\mathrm{SnTe})_{1-y}\left(\mathrm{Sb}_{2} \mathrm{Se}_{3}\right)_{y}$ synthesized via solvothermal reaction and sintering. ACS Appl Mater Interfaces 2020, 12: 44805-44814.

[22] Zhang YM, Shen XC, Yan YC, et al. Enhanced thermoelectric performance of ternary compound $\mathrm{Cu}_{3} \mathrm{PSe}_{4}$ by defect engineering. Rare Metal 2020, 39: 1256-1261.

[23] Guo FK, Cui B, Liu Y, et al. Thermoelectric SnTe with band convergence, dense dislocations, and interstitials through Sn self-compensation and Mn alloying. Small 2018, 14: 1802615.

[24] Tan X, Lan JL, Liu YC, et al. Optimization of the thermoelectric properties of $\mathrm{Bi}_{2} \mathrm{O}_{2} \mathrm{Se}$ ceramics by altering the temperature of spark plasma sintering. $J$ Electroceramics 2016, 37: 66-72.

[25] Shen XC, Zhang X, Zhang B, et al. Optimizing thermoelectric properties of $\mathrm{BiSe}$ through $\mathrm{Cu}$ additive enhanced effective mass and phonon scattering. Rare Metal 2020, 39: 1374-1382.
[26] Rowe DM, Bhandari CM. Lattice thermal conductivity of small grain size $\mathrm{PbSnTe}$ and $\mathrm{PbGeTe}$ thermoelectric material. Appl Phys Lett 1985, 47: 255-257.

[27] Wu D, Chen X, Zheng FS, et al. Dislocation evolution and migration at grain boundaries in thermoelectric SnTe. ACS Appl Energy Mater 2019, 2: 2392-2397.

[28] Tan GJ, Zhao LD, Shi FY, et al. High thermoelectric performance of p-type SnTe via a synergistic band engineering and nanostructuring approach. $\mathrm{J} \mathrm{Am} \mathrm{Chem} \mathrm{Soc}$ 2014, 136: 7006-7017.

[29] Acharya S, Pandey J, Soni A. Soft phonon modes driven reduced thermal conductivity in self-compensated $\mathrm{Sn}_{1.03} \mathrm{Te}$ with Mn doping. Appl Phys Lett 2016, 109: 133904.

[30] He J, Tan XJ, Xu JT, et al. Valence band engineering and thermoelectric performance optimization in SnTe by Mn-alloying via a zone-melting method. J Mater Chem A 2015, 3: 19974-19979.

[31] Guo FK, Cui B, Geng HY, et al. Simultaneous boost of power factor and figure-of-merit in in-Cu codoped SnTe. Small 2019, 15: 1902493.

[32] Sarkar D, Ghosh T, Banik A, et al. Highly converged valence bands and ultralow lattice thermal conductivity for high-performance SnTe thermoelectrics. Angew Chem Int Ed 2020, 59: 11115-11122.

[33] Chen ZW, Jian ZZ, Li W, et al. Lattice dislocations enhancing thermoelectric $\mathrm{PbTe}$ in addition to band convergence. Adv Mater 2017, 29: 1606768.

[34] Wu YX, Chen ZW, Nan PF, et al. Lattice strain advances thermoelectrics. Joule 2019, 3: 1276-1288.

[35] Hong M, Wang Y, Xu SD, et al. Nanoscale pores plus precipitates rendering high-performance thermoelectric $\mathrm{SnTe}_{1-x} \mathrm{Se}_{x}$ with refined band structures. Nano Energy 2019, 60: 1-7.

[36] Zong PA, Hanus R, Dylla M, et al. Skutterudite with graphene-modified grain-boundary complexion enhances $z T$ enabling high-efficiency thermoelectric device. Energy Environ Sci 2017, 10: 183-191.

[37] Wu D, Chen X, Zheng FS, et al. Dislocation evolution and migration at grain boundaries in thermoelectric SnTe. ACS Appl Energy Mater 2019, 2: 2392-2397.

[38] Li J, Zhang XY, Lin SQ, et al. Realizing the high thermoelectric performance of GeTe by Sb-doping and Se-alloying. Chem Mater 2017, 29: 605-611.

[39] Zhang JW, Wu ZW, Xiang B, et al. Ultralow lattice thermal conductivity in SnTe by incorporating InSb. ACS Appl Mater Interfaces 2020, 12: 21863-21870.

[40] Dong JF, Wu CF, Pei J, et al. Lead-free MnTe mid-temperature thermoelectric materials: Facile synthesis, p-type doping and transport properties. J Mater Chem C 2018, 6: 4265-4272.

[41] Marques MAL, Vidal J, Oliveira MJT, et al. Density-based mixing parameter for hybrid functionals. Phys Rev B 2011, 83: 035119.

[42] Blöchl PE. Projector augmented-wave method. Phys Rev B 1994, 50: 17953. 
[43] Perdew JP, Burke K, Ernzerhof M. Generalized gradient approximation made simple. Phys Rev Lett 1996, 77 : 3865-3868.

[44] Xiao Y, Wu HJ, Li W, et al. Remarkable roles of $\mathrm{Cu}$ to synergistically optimize phonon and carrier transport in

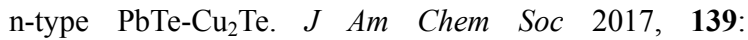
18732-18738.

[45] Liu CY, Huang ZW, Wang DH, et al. Dynamic $\mathrm{Ag}^{+}-$ intercalation with $\mathrm{AgSnSe}_{2}$ nano-precipitates in Cl-doped polycrystalline $\mathrm{SnSe}_{2}$ toward ultra-high thermoelectric performance. J Mater Chem A 2019, 7: 9761-9772.

[46] Zhang Q, Liao B, Lan Y, et al. High thermoelectric performance by resonant dopant indium in nanostructured SnTe. PNAS 2013, 110: 13261-13266.

[47] Brebrick RF, Strauss AJ. Anomalous thermoelectric power as evidence for two-valence bands in SnTe. Phys Rev 1963, 131: 104.

[48] Tan GJ, Shi FY, Doak JW, et al. Extraordinary role of $\mathrm{Hg}$ in enhancing the thermoelectric performance of p-type SnTe. Energy Environ Sci 2015, 8: 267-277.

[49] Pei YZ, Zheng LL, Li W, et al. Interstitial point defect scattering contributing to high thermoelectric performance in SnTe. Adv Electron Mater 2016, 2: 1600019.

[50] Johnsen S, He JQ, Androulakis J, et al. Nanostructures boost the thermoelectric performance of PbS. J Am Chem
Soc 2011, 133: 3460-3470.

[51] Kim HS, Kang SD, Tang YL, et al. Dislocation strain as the mechanism of phonon scattering at grain boundaries. Mater Horiz 2016, 3: 234-240.

[52] Pan Y, Aydemir U, Grovogui JA, et al. Melt-centrifuged $(\mathrm{Bi}, \mathrm{Sb})_{2} \mathrm{Te}_{3}$ : Engineering microstructure toward high thermoelectric efficiency. Adv Mater 2018, 30: 1802016.

[53] Mion C, Muth JF, Preble EA, et al. Thermal conductivity, dislocation density and GaN device design. Superlattices Microstruct 2006, 40: 338-342.

Open Access This article is licensed under a Creative Commons Attribution 4.0 International License, which permits use, sharing, adaptation, distribution and reproduction in any medium or format, as long as you give appropriate credit to the original author(s) and the source, provide a link to the Creative Commons licence, and indicate if changes were made.

The images or other third party material in this article are included in the article's Creative Commons licence, unless indicated otherwise in a credit line to the material. If material is not included in the article's Creative Commons licence and your intended use is not permitted by statutory regulation or exceeds the permitted use, you will need to obtain permission directly from the copyright holder.

To view a copy of this licence, visit http://creativecommons.org/licenses/by/4.0/. 